\title{
The role of rehabilitative camouflage after cervicofacial reconstructive surgery: a preliminary study
}

This article was published in the following Dove Press journal:

Clinical, Cosmetic and Investigational Dermatology

30 January 2014

Number of times this article has been viewed

\author{
Giovanni Nicoletti ${ }^{1-3}$ \\ Andrea Sasso' \\ Alberto Malovini ${ }^{4,5}$ \\ Luisa Ponchio ${ }^{6}$ \\ Silvia Scevola ${ }^{2}$ \\ Angela Faga ${ }^{1-3}$ \\ Aldo Pontone'
}

'Plastic and Reconstructive Surgery, Department of Clinical Surgical Diagnostic and Pediatric Sciences, ${ }^{2}$ Advanced Technologies for Regenerative Medicine and Inductive Surgery Research Centre, University of Pavia, Pavia, Italy; ${ }^{3}$ Plastic and Reconstructive Surgery Unit, Salvatore Maugeri Research and Care Institute, Pavia, Italy; ${ }^{4}$ Department of Computer Engineering and Systems Science, University of Pavia, Pavia, Italy; ${ }^{5}$ Laboratory of Informatics and Systems Engineering for Clinical Research, ${ }^{6}$ Oncology Unit, Salvatore Maugeri Research and Care Institute, Pavia, Italy
Correspondence: Giovanni Nicoletti Plastic Surgery Unit, University of Pavia, Salvatore Maugeri Research and Care Institute, Via Salvatore Maugeri I0,

27I00 Pavia, Italy

Tel +390382 592 222;

+393385778146 (mobile)

Fax +390382592220

Email giovanni.nicoletti@unipv.it; giovanni.nicoletti@fsm.it
Abstract: A randomized, prospective, controlled study was carried out at the Plastic and Reconstructive Surgery Unit of the University of Pavia, Salvatore Maugeri Research and Care Institute, Pavia, Italy, to evaluate the psychological benefits from corrective medical camouflage (CMC) following surgical treatment for skin cancer of the face. Twenty-four female patients, following recovery from facial skin cancer surgery, were enrolled in the study over a period of 1 year. The study was performed using two health-related quality of life tests, the Satisfaction Profile (SAT-P) test and the Body Uneasiness Test (BUT). The patients were randomized into two groups: group A, patients undergoing CMC; and group B, controls. Both the SAT-P and BUT demonstrated statistically significant better results in the treated patients versus the controls in the following functional parameters: Psychological Functionality (PsF), Physical Functionality (PhF), and Work Performance (WP) for the SAT-P test and Compulsive SelfMonitoring (CSM) for the BUT. The PsF demonstrated a better result 6 months post-treatment. Such a difference was particularly significant when comparing the performance at 6 months versus that at 3 months. The $\mathrm{PhF}$ demonstrated a better outcome at 6 months post-treatment. The WP demonstrated a better result comparing the performance at 6 months versus that at 3 months. The CSM demonstrated a better outcome at 6 months post-treatment. The CMC promoted a significant improvement in patients' physical appearance and in their self-image and perceived social role as a means of their desire to disguise their body disfiguration.

Keywords: plastic surgery, skin cancer, camouflage, quality of life, psychological assessment

\section{Introduction}

Disfiguring scars following surgery are known to give rise to a wide range of psychological well-being changes in patients. ${ }^{1}$ In general, exposed scars, and particularly on those body areas prominently used in social interaction situations, may be source of concern even after a technically perfect reconstruction. ${ }^{2}$ Head and neck skin tumors may require wide and disabling surgical excisions in order to achieve significant healing rates. Disfigurement is sometimes the unavoidable consequence following surgical treatment of such potentially lethal diseases. ${ }^{3}$ Concern about self-image loss is often coupled with the perceived fear of cancer and leads to a continuously stressful condition that may persist well after healing. ${ }^{4}$ Regarding plastic surgery, the patients' subjective expectations are often highly unrealistic: the objective technical limitations in providing aesthetically pleasing reconstructions support patients' dissatisfaction, and may lead to the markings of medicolegal litigations. ${ }^{5}$

It is a well-known fact that corrective medical camouflage (CMC) may improve the psychological condition of patients suffering from chronic skin diseases or undergoing 
chemotherapy for cancer; such benefits are also demonstrated postoperatively in patients undergoing aesthetic surgery. ${ }^{6-10}$ An improvement in the perceived self-image of these patients ultimately leads to significant stress reduction.

The aim of our study was the objective evaluation of the psychological benefits from CMC following surgical treatment for skin cancer of the face, using the original integration of two validated tools for health-related quality of life assessment.

\section{Methods}

A randomized, prospective, controlled study was carried out at the Plastic and Reconstructive Surgery Unit of the University of Pavia, Salvatore Maugeri Research and Care Institute, Pavia, Italy.

In order to provide the most homogeneous sample, the trial involved female patients only.

Twenty-four women following stable and uneventful recovery from surgical treatment for skin cancer of the face were enrolled in the study over a period of 1 year, from September 2011 to September 2012. The mean age was 67.12 years (minimum 53, maximum 80, median 67).

Exclusion criteria were current or past psychiatric comorbidities and/or history of psychotherapy. The patients were randomized using a computerized random number generator into two groups of 12 individuals: group A comprised patients undergoing $\mathrm{CMC}$ and group B was the control group.

The cosmetic products were produced and provided by Istituto Ganassini Corporate, Milan, Italy. The manufacturer guaranteed noncomedogenic, hypoallergenic, and microbiologically and dermatologically tested formulas.

The original integration of two popular and reliable health-related quality of life psychometric tests was used in our study: the Satisfaction Profile (SAT-P) test, which provides both a qualitative and quantitative self-satisfaction assessment within the health-related quality of life; ${ }^{11}$ and the Body Uneasiness Test (BUT), which allows a psychometric evaluation of self-image and body-image uneasiness. ${ }^{12}$ The tests were conducted by a single psychologist. Both groups of patients were assessed 30 to 60 days postoperatively $\left(\mathrm{T}_{0}\right)$, before undergoing $\mathrm{CMC}$, and at $3\left(\mathrm{~T}_{1}\right)$ and $6\left(\mathrm{~T}_{2}\right)$ months post-treatment.

The patients in group A attended three CMC training sessions. Each session lasted about 2 hours, was attended by four patients, and took place in a comfortably furnished room. A large table was placed in the middle of the room and each patient was given a mirror and makeup products. A professional makeup artist prepared customized CMC designs that were submitted for patient approval. The patients were then trained to reproduce the makeup in everyday life. At the end of each session, the psychologist wrote up a comprehensive observational report.

The trial was approved on July 21, 2011 by the Salvatore Maugeri Research and Care Institute ethical committee (protocol number 730).

\section{Statistical analysis}

Variations in terms of score distributions were estimated as the difference in terms of individual level values at different time points $\left(\right.$ delta $_{t 0-t 1}=\mathrm{T}_{1}-\mathrm{T}_{0}$; delta $_{t 0-t 2}=\mathrm{T}_{2}-\mathrm{T}_{0}$; and delta $\left._{t 1-t 2}=\mathrm{T}_{2}-\mathrm{T}_{1}\right)$. Differences in terms of score variations (delta values) between cases and controls were tested by the two-sided Wilcoxon rank-sum test. Permutation tests were applied for adjusting statistics for multiple comparisons: a total number of 1,000 permutations were performed for each test. Distributions are expressed in terms of median and interquartile range $\left(25^{\text {th }}: 75^{\text {th }}\right.$ percentiles $)$. Associations were deemed statistically significant when both unadjusted and permutation adjusted $P$-values were $<0.05$. Statistical procedures were performed using $\mathrm{R}$ software, version 3.0, 2 (The R Foundation for Statistical Computing, Vienna, Austria).

\section{Results}

Association results are reported in Tables 1 and 2.

The SAT-P test demonstrated a statistically significant variation in the scores of the following functional parameters: Psychological Functionality (PsF) (delta ${ }_{t 0-t 2}$ and delta $\left._{t 1-t 2}\right)$; Physical Functionality (PhF) $\left(\right.$ delta $\left._{t 0-t 2}\right)$; and Work Performance (WP) $\left(\right.$ delta $\left._{t 1-t 2}\right)$. In the BUT, a statistically significant variation was demonstrated in the Compulsive Self-Monitoring (CSM) scores ( delta $\left._{t 0-t 2}\right)$ (Figure 1).

None of the investigated functional parameters demonstrated statistically significant variations at 3 months post-treatment. The statistically significant favorable results were observed at 6 months post-treatment.

PsF demonstrated a statistically significant variation in the treated patients versus the controls at 6 months post-treatment $(P=0.004)$. In detail, such a difference was particularly significant when comparing the performance at 6 months versus that at 3 months $(P=0.003)$.

$\mathrm{PhF}$ also demonstrated a statistically significant variation in the treated patients versus the controls at 6 months posttreatment $(P=0.031)$; nevertheless, the difference between the performance at 6 months and that at 3 months was not statistically significant. 

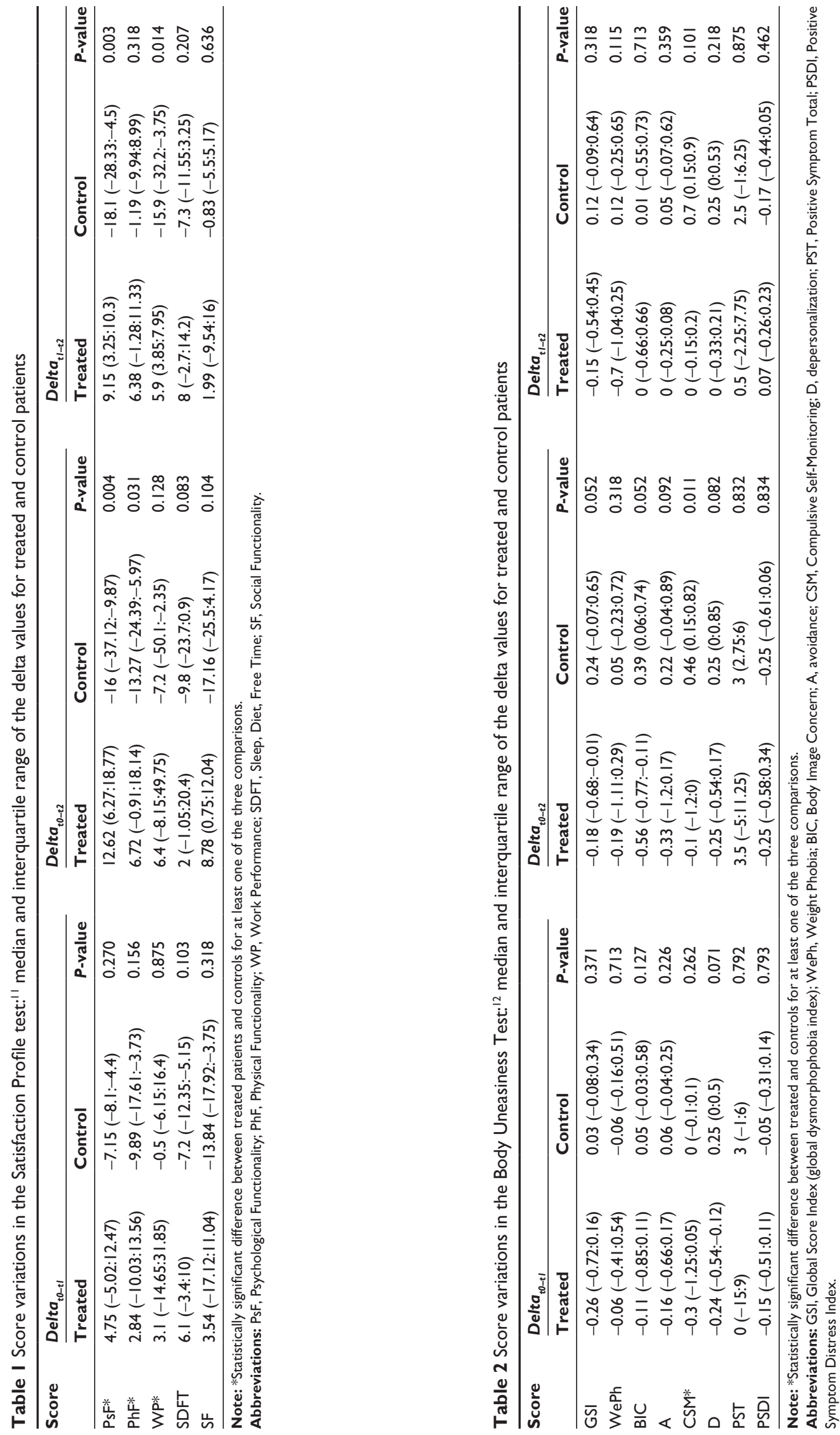

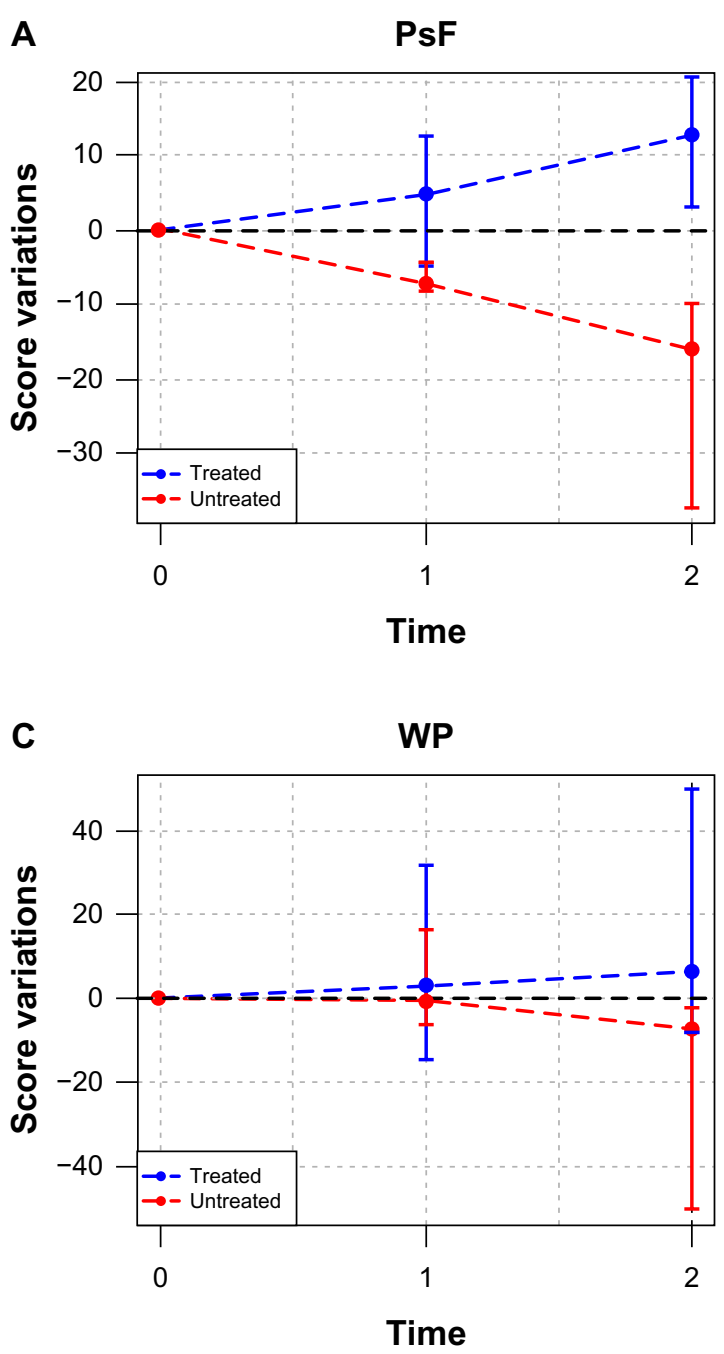

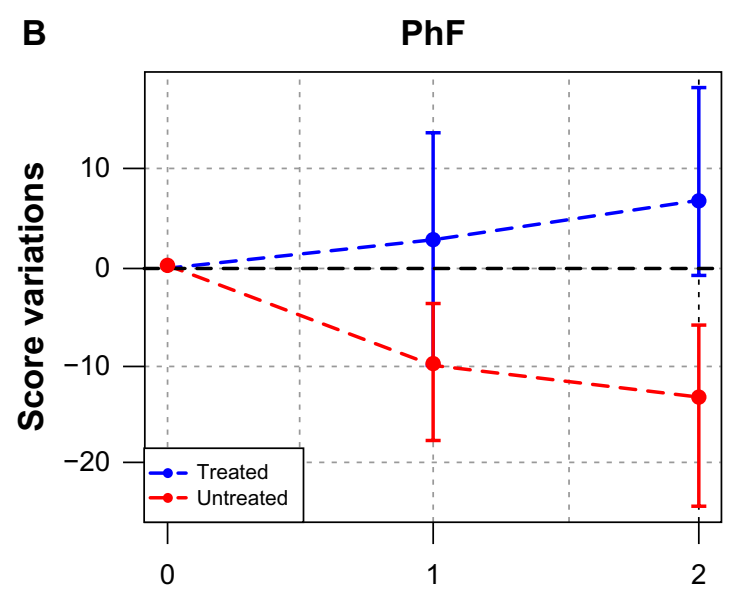

Time

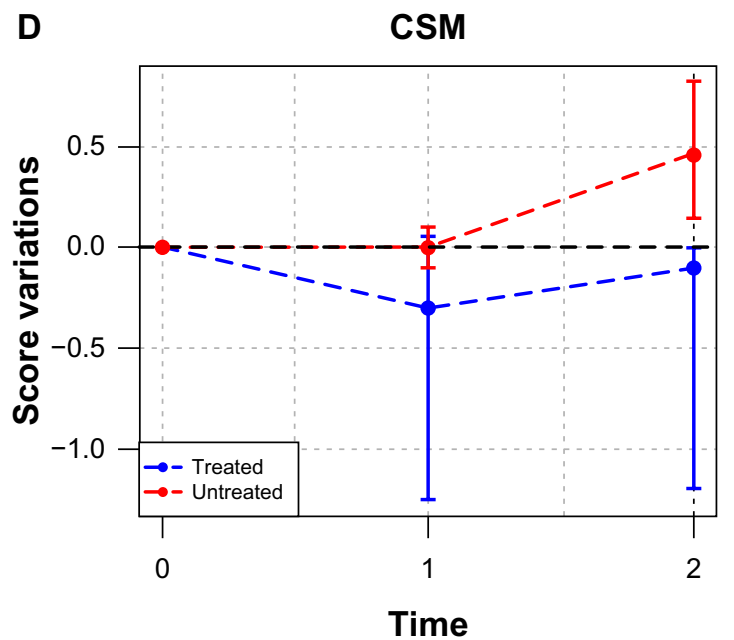

Figure I Statistically significant score variations for patients undergoing corrective medical camouflage and controls.

Notes: (A) Psychological Functionality; (B) Physical Functionality; (C) Work Performance; (D) Compulsive Self-Monitoring. Points and horizontal bars represent the median value and interquartile range of the delta scores, respectively. Time points: $0=30$ to 60 days postoperatively; I=3 months post-treatment; $2=6$ months post-treatment. Abbreviations: PsF, Psychological Functionality; PhF, Physical Functionality; WP, Work Performance; CSM, Compulsive Self-Monitoring.

WP demonstrated a statistically significant variation in the treated patients versus the controls, comparing the performance at 6 months versus that at 3 months $(P=0.014)$. A statistically significant variation was not demonstrated, both at 3 months post-operatively vs pre-operatively, and at 6 months postoperatively vs pre-operatively; the only statistically significant variation was at at 6 months post-operatively vs 3 months post-operatively, as stated in the former period.

CSM demonstrated a statistically significant variation in the treated patients versus the controls at 6 months post-treatment $(P=0.011)$, although the difference between the performance at 6 months and that at 3 months was not statistically significant.

\section{Discussion}

There is currently no gold standard available among the huge variety of tools used to assess the impact of appearance changes in head and neck cancer patients. There are several reports in the literature that assess quality of life in head and neck cancer patients, but none have reviewed instruments that specifically assess patients' appearance satisfaction. ${ }^{13-18}$ In our study, we integrated two popular and reliable health-related quality of life psychometric tests: the SAT-P test and the BUT, the latter having previously been validated for the preoperative assessment of patients undergoing aesthetic surgery procedures, which to our knowledge have not been integrated before. ${ }^{19}$

Within the overall investigated functional parameters, only four demonstrated a statistically significant variation in the treated patients versus the controls: PsF, PhF, WP, and CSM.

Among these parameters, PsF showed the most consistent improvement ( delta $_{t 0-t 2} P=0.004 ;$ delta $_{t 1-t 2} P=0.003$ ). Such an improvement allows for an increase of both self-satisfaction and psychological well-being within the following individual and social scopes: setting oneself a goal, a mission, and a vision in life; personal development; maintaining good 
relationship with other people, whether family members or not; control ability and sense of personal self-efficacy; self-acceptance, self-respect, and self-esteem; psychological autonomy; and personal reflection ability. The parameter PsF also clearly defines the subjective perception of illness, thus providing a precise estimate of what, in the patient's perception, has been diminished by their disease. Nevertheless, this parameter allows an accurate assessment of the positive effects of treatment on a patient's life, such as recovering one's values, the consolidation of effective relationships, and the development of new and unexpected abilities. All of these remarkable improvements are usually not appreciated in the short term, but tend to undoubtedly and suddenly appear in the second trimester of treatment.

The PhF parameter demonstrated a statistically significant difference in the treated patients versus the controls at 6 months' follow-up ( delta $_{t 0-t 2} P=0.031$ ). According to this parameter, $\mathrm{CMC}$ could reduce the self-induced withdrawal from active life promoted by feeling "sick" or "disabled", thereby promoting a sensation of full physical well-being. The PhF parameter comprehensively investigates overall physical well-being, sleep quality, and appetite. Failure in demonstrating any statistically significant difference in the score variation of this parameter between 3 and 6 months posttreatment would suggest a slow and progressive improvement that may take at least 6 months to be seen clearly.

WP failed to demonstrate a significant improvement in the group of patients undergoing $\mathrm{CMC}$ versus the controls both at 3 and 6 months' follow-up. Nevertheless, a statistically significant delta could be appreciated between the outcomes at 3 and 6 months post-treatment, thus suggesting a late and slow onset of the benefits in the second trimester of treatment. The WP parameter is able to comprehensively assess the working efficiency, the perceived work satisfaction, and one's working identity and everyday life abilities. All of these aspects are of great importance, both in the individual's general recovery and in obtaining a stable, disease-free remission.

CSM demonstrated a statistically significant difference in the treated patients versus the controls at 6 months' follow-up $\left(\right.$ delta $\left._{t 0-t 2} P=0.011\right)$. A compulsive and obsessive concern for perceived self image usually increases in the patient suffering from oncological disease every time social interaction requires body parts that may reveal a past surgical treatment to be visibly exposed. CMC could therefore counterbalance such diseaserelated emotional reactions and shift the patient's attention from self-commiseration to a more positive outlook. There is also a consequent improvement in relative concerns regarding self-image. Failure to demonstrate any statistically significant difference in the score variation of this parameter between 3 and
6 months post-treatment would suggest a slow and progressive improvement that may take up to 6 months to show results.

CMC allowed for a significant improvement in the skin color match on the operated face and a favorable alteration of the facial light points, with apparent rebalance of facial volume (Figure 2). Excellent color matching of scars was achieved, making the scars inconspicuous. Facial asymmetry became less evident, and all skin imperfections could be disguised.

Scars in general tend to improve with time, and visible improvements could be appreciated in the control group after 6 months (Figure 3). Nevertheless, our results demonstrated a significant advantage in the treated patients versus the controls in the perception of their overall quality of life.

CMC promoted a significant improvement both in patients' physical appearance and in their self-image and perceived social role due to disguising of body disfigurement. Before treatment, patients had poor self-image and overwhelming social withdrawal induced by the physical illness. The group of patients undergoing CMC experienced a strong recovery of their self-image, with significant improvement of all the vital functional parameters, and self-satisfaction. Their physical appearance no longer gave rise to negative emotions, but instead inspired the patients to take more care of themselves, coupled with feelings of being accepted within social interactions. We believe that CMC could shift patients' attention from their continuously perceived illness to a more positive attitude, where their perceived self-image is fully and harmoniously reintegrated into their emotional life. A key role in recovering such a psychological balance is played out by the close interaction between the desire for improved appearance and improved social relationships. This was demonstrated by the significant reduction of the patient's compulsive control of their physical appearance and the increase of the work related satisfaction.
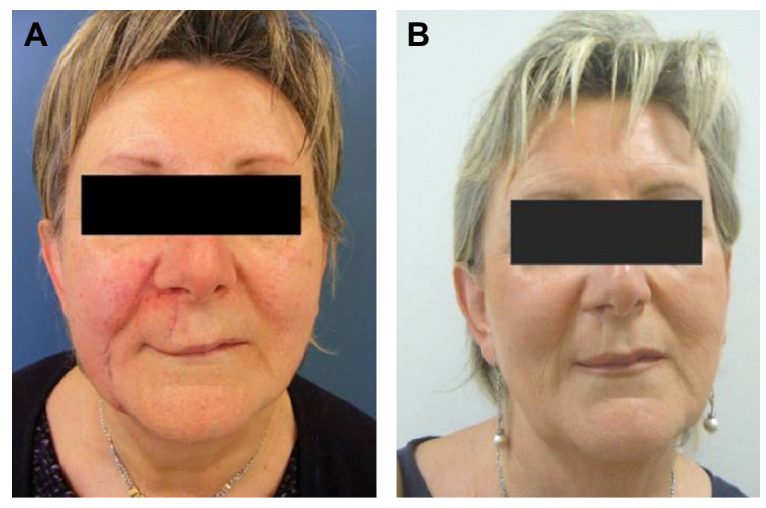

Figure 2 Pre- and post-treatment comparative images.

Notes: (A) Result after excision of basal cell carcinoma of the right upper lip and reconstruction with local skin rotation flap. Erythematous scars and upper lip asymmetry are visible. (B) The same patient after corrective medical camouflage. 

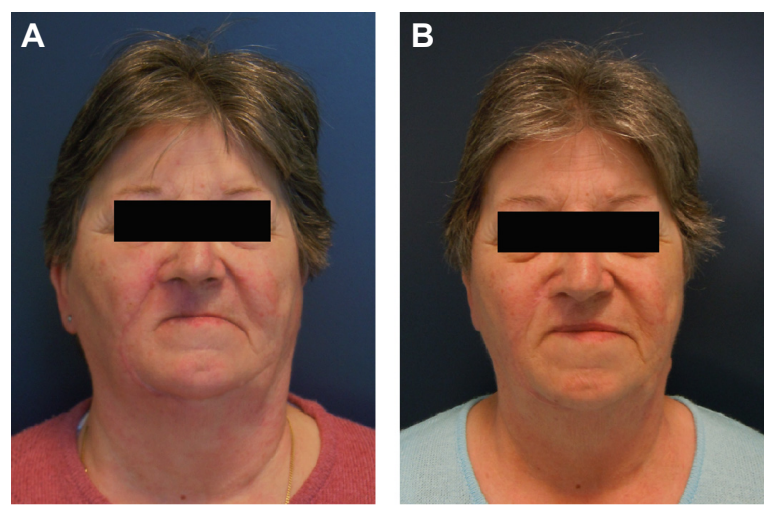

Figure 3 Control patient postoperatively and at 6 months' follow-up. Notes: (A) The patient after excision of basal cell carcinoma of the right upper lip and reconstruction with local skin rotation flap. Erythematous scars and upper lip asymmetry are visible. (B) The same patient at 6 months' follow-up.

Undoubtedly, our study suffered from several shortcomings, such as the small group of patients; the different defects in different patients, even though they were all facial; and the short follow-up time. We believe that a larger sample and a longer follow-up would likely demonstrate further benefits of CMC.

On the other hand, in our experience, group therapy with such a small number of participants allowed the patients to benefit from sharing their experiences of their self-actualizing needs and feelings of social isolation. Consequently, this offered a pathway to alleviate associated stresses due to their conditions.

Our study involved female patients only, as this demographic tends to habitually use makeup. We actually do not know how men would respond to this form of therapy, as men are generally less prone to wearing makeup. Actually, the adverse attitudes of men in western culture toward makeup use is related to the contemporary historical context, although, in the European culture, males used to wear makeup in past times. In modern times, men usually only wear make-up in non-Western cultures. Nevertheless, there seems to be a recent cultural trend featuring an increasingly favorable attitude of men toward cosmetic care in western society; as such, male prejudice against makeup might be overcome in the not-too-distant future.

No adverse toxic or allergic reactions to the cosmetic products used in the study were observed.

The minor overall cost of CMC proved to be highly convenient and cost-effective in terms of quality-of-life benefits.

\section{Conclusion}

This study demonstrates the great contribution of a simple and, to some, superficial procedure that can both help the patient escape the anguish of disfiguration and reduce rancor against the surgeon, who is often blamed for devastating a patient's self-image. CMC definitely proved to be a worthwhile refinement in the process of functional and cosmetic post-oncological reconstruction, and our results would support the postoperative involvement of a beauty specialist nurse among plastic surgery unit staff.

\section{Acknowledgments}

The costs of the tools, cosmetic products, and makeup artist were granted by Istituto Ganassini Corporate, Milan, Italy. The authors wish to thank Francesco Martina, professional makeup artist, for his substantial contribution to the study. The authors also wish to thank Alan Serge McGhee M.Sc, Glasgow City Council Education Department, for his contribution to the submission of this dissertation.

\section{Disclosure}

The authors report no conflicts of interest in this work.

\section{References}

1. Rankin M, Borah G. Psychological complications: national plastic surgical nursing survey. Plast Surg Nurs. 2009;29(1):25-30; quiz 31-32.

2. Borah G, Rankin M, Wey P. Psychological complications in 281 plastic surgery practices. Plast Reconstr Surg. 1999;104(5):1241-1246.

3. Moolenburgh SE, Mureau MA, Versnel SL, Duivenvoorden HJ, Hofer SO. The impact of nasal reconstruction following tumor resection on psychosocial functioning, a clinical-empirical exploration. Psychooncology. 2009;18(7):747-752.

4. Ganz PA, Coscarelli A, Fred C, Kahn B, Polinsky ML, Petersen L. Breast cancer survivors: psychosocial concerns and quality of life. Breast Cancer Res Treat. 1996;38(2):183-199.

5. Rankin M, Borah GL. Anxiety disorders in plastic surgery. Plast Reconstr Surg. 1997;100(2):535-542.

6. Erdos D. Look Good ... Feel Better - a program for cancer patients. $R N .1992 ; 55(10): 25-26,28$.

7. Taggart LR, Ozolins L, Hardie H, Nyhof-Young J. Look good feel better workshops: a "big lift" for women with cancer. J Cancer Educ. 2009;24(2):94-99.

8. Sidle DM, Decker JR. Use of makeup, hairstyles, glasses, and prosthetics as adjuncts to scar camouflage. Facial Plast Surg Clin North Am. 2011;19(3):481-489.

9. Aydogdu E, Misirlioglu A, Eker G, Aköz T. Postoperative camouflage therapy in facial aesthetic surgery. Aesthetic Plast Surg. 2005;29(3): 190-194.

10. Holme SA, Beattie PE, Fleming CJ. Cosmetic camouflage advice improves quality of life. Br J Dermatol. 2002;147(5):946-949.

11. Majani G, Callegari S. SAT-P Satisfaction Profile. Soddisfazione Soggettiva e Qualità Della Vita [SAT-P Satisfaction Profile. Subjective Satisfaction and Quality of Life]. Trento: Centro Studi Erickson; 2003. Italian.

12. Cuzzolaro M, Vetrone G, Marano G, Garfinkel PE. The Body Uneasiness Test (BUT): development and validation of a new body image assessment scale. Eat Weight Disord. 2006;11(1):1-13.

13. Djan R, Penington A. A systematic review of questionnaires to measure the impact of appearance on quality of life for head and neck cancer patients. J Plast Reconstr Aesthet Surg. 2013;66(5):647-659.

14. Hassan SJ, Weymuller EA Jr. Assessment of quality of life in head and neck cancer patients. Head Neck. 1993;15(6):485-496.

15. Ringash J, Bezjak A. A structured review of quality of life instruments for head and neck cancer patients. Head Neck. 2001;23(3):201-213. 
16. Gliklich RE, Goldsmith TA, Funk GF. Are head and neck specific quality of life measures necessary? Head Neck. 1997;19(6):474-480.

17. Klassen A, Jenkinson C, Fitzpatrick R, Goodacre T. Measuring quality of life in cosmetic surgery patients with a condition-specific instrument: the Derriford Scale. Br J Plast Surg. 1998;51(5):380-384.
18. Harris D, Moss TP, Carr T. Manual for the Derriford Appearance Scale 59 (DAS59). Bradford on Avon: Musketeer Press; 2004.

19. Pollice R, Giuliani M, Bianchini V, et al. [Body image disorders and other psychiatric symptoms in aesthetic plastic surgery]. Ann Ital Chir. 2009;80:49-54. Italian.

\section{Publish your work in this journal}

Clinical, Cosmetic and Investigational Dermatology is an international, peer-reviewed, open access, online journal that focuses on the latest clinical and experimental research in all aspects of skin disease and cosmetic interventions. All areas of dermatology will be covered; contributions will be welcomed from all clinicians and basic science researchers globally. This journal is indexed on CAS. The manuscript management system is completely online and includes a very quick and fair peer-review system, which is all easy to use. Visit http://www.dovepress.com/testimonials.php to read real quotes from published authors.

Submit your manuscript here: http://www.dovepress.com/clinical-cosmetic-and-investigational-dermatology-journal 